\title{
CORRECTION
}

Check for updates

Cite this: Nanoscale Horiz., 2020, 5,373

\section{Correction: Engineering spin and antiferromagnetic resonances to realize an efficient direction-multiplexed visible meta-hologram}

\author{
Muhammad Afnan Ansari, ab Inki Kim, ${ }^{c}$ Ivan D. Rukhlenko, de Muhammad Zubair, ${ }^{a}$ \\ Selcuk Yerci, ${ }^{\text {bfg }}$ Tauseef Tauqeer, ${ }^{* a}$ Muhammad Qasim Mehmood*a and \\ Junsuk Rho*chi
}

DOI: $10.1039 / \mathrm{dOnh} 90006 \mathrm{k}$

Correction for 'Engineering spin and antiferromagnetic resonances to realize an efficient direction-

rsc.li/nanoscale-horizons

The authors regret that a funding source was omitted in the 'Acknowledgements' section of the original article. A revised version of the 'Acknowledgements' section, adding the funding received from the National Research Foundation of Korea under grant number NRF-2019R1A5A8080290, is provided below.

The Royal Society of Chemistry apologises for these errors and any consequent inconvenience to authors and readers.

\section{Acknowledgements}

This work was supported by the LGD-SNU incubation program and the National Research Foundation of Korea (NRF) grants (NRF2019R1A2C3003129, CAMM-2019M3A6B3030637，NRF-2018M3D1A1058998，NRF-2015R1A5A1037668，NRF-2019R1A5A8080290) funded by the Ministry of Science and ICT (MSIT) of the Korean government. This work was also supported by the Government of the Russian Federation through the ITMO Fellowship and Professorship Program. The work was also supported by Higher Education Commission of Pakistan (Award No. 10177). M. A. A. acknowledges the Scientific and Technological Research Council of Turkey (TÜBITAK) for the research grant "2216 - Research Scholarship for International Researchers (Award No. B.14.2.TBT.0.06.01.02-216-10539)". M. Q. M., T. T., and M. Z. acknowledge an internal research grant from ITU. I. K. acknowledges the Global PhD fellowship (NRF-2016H1A2A1906519) by NRF-MIST of the Korean government. I. R. gratefully acknowledges the financial support from the Russian Science Foundation (RSF) (Grant No. 19-13-00332).

\footnotetext{
${ }^{a}$ NanoTech Lab, Department of Electrical Engineering, Information Technology University of the Punjab, Lahore 54600, Pakistan. E-mail: qasim.mehmood@itu.edu.pk, tauseef.tauqeer@itu.edu.pk

${ }^{b}$ Department of Electrical and Electronics Engineering, Middle East Technical University, Cankaya/Ankara 06800, Turkey. E-mail: syerci@metu.edu.tr

${ }^{c}$ Department of Mechanical Engineering, Pohang University of Science and Technology (POSTECH), Pohang, 37673, Republic of Korea. E-mail: jsrho@postech.ac.kr

${ }^{d}$ Information Optical Technologies Centre, ITMO University, Saint Petersburg 197101, Russia

${ }^{e}$ Institute of Photonics and Optical Sciences (IPOS), School of Physics, The University of Sydney, Camperdown 2006, New South Wales, Australia

${ }^{f}$ Department of Micro and Nanotechnology, Middle East Technical University, 06800 Çankaya/Ankara, Turkey

${ }^{g}$ Center for Solar Energy Research and Applications, Middle East Technical University, 06800 Çankaya/Ankara, Turkey

${ }^{h}$ Department of Chemical Engineering, Pohang University of Science and Technology (POSTECH), Pohang 37673, Republic of Korea

${ }^{i}$ National Institute of Nanomaterials Technology (NINT), Pohang 37673, Republic of Korea
} 